\title{
The effect of radiation on transient natural convection past a doubly infinite plate
}

\author{
I. Pop, Cluj and T. Y. Na, Michigan
}

\begin{abstract}
The present research note is concerned with the transient (short time) simultaneous free convection and radiation analysis of a viscous fluid along a doubly infinite vertical isothermal flat plate. To simplify a very complicated problem, an incompressible flow field is used in the analysis.

Generally, the exact numerical solution of this problem is quite lengthy. However, by considering an optically thick radiating gas, expressed by the Rosseland diffusion approximation, the solution is much simpler. Moreover, this case leads to a complete similarity transformation of the governing partial differential equations into a set of ordinary differential equations. An exact numerical solution is obtained of the resulting ordinary differential equations for a Prandtl number equal to 0.733 and for a wide range of involved parameters.
\end{abstract}

\section{Der Einfluß der Strahlung auf transiente freie Konvektion längs einer unendlichen Platte}

Zusammenfassung. Der vorliegende Bericht befaßt sich mit der Untersuchung der transienten Vorgänge bei gleichzeitiger freier Konvektion und Strahlung an einem zähen Fluid entlang einer unendlichen isothermen ebenen Platte. Um das komplizierte Problem zu vereinfachen, wird eine inkompressible Strömung angenommen.

Normalerweise ist die exakte numerische Lösung dieses Problems sehr langwierig. Berücksichtigt man jedoch ein optisch dichtes, strahlendes Gas wie es durch die Rosselandsche Diffusionsnäherung ausgedrückt wird, ist die Lösung wesentlich einfacher. Zudem führt dieser Fall zu einer kompletten Ähnlichkeitstransformation der beschreibenden partiellen Differentialgleichungen in einen Satz von gewöhnlichen Differentialgleichungen. Eine exakte numerische Lösung wird erzielt für eine PrandtlZahl von 0.733 und einem großen Bereich der beteiligten Parameter.

\section{Introduction}

There is a considerable amount of work in the literature on the combined conduction and radiation heat transfer in a semi-infinite medium with variable thermophysical and radiative properties. In particular, the unsteady laminar boundary layer flow past an infinite flat plate and near a three-dimensional stagnation point with radiative heat transfer have been considered in [1,2]. But, there appears to be no analysis of radiative heat transfer effects on unsteady free convection flow past vertical or horizontal flat plates. The present research note is therefore con- cerned with the transient (short time) simultaneous free convection and radiation analysis of a viscous fluid along a doubly infinite vertical isothermal flat plate. To simplify, otherwise, a very complicated problem, an incompressible flow field is used in the analysis.

Generally, the exact numerical solution of this problem is quite lengthy. However, by considering an optically thick radiating gas, expressed by Rosseland diffusion approximation [3], the solution is much simpler. Moreover, this case leads to a complete similarity transformation of the governing partial differential equations into a set of ordinary differential equations. An exact numerical solution is obtained of the resulting ordinary differential equations for a Prandtl number equal to 0.733 and for a wide range of involved parameters.

\section{Basic equations}

Let be a vertical, doubly infinite flat plate immersed in an absorbing and emitting gas subject to the following assumptions: (i) the flow is laminar and in unsteady state, (ii) the properties of the gas are constant; this also implies the gas is gray, (iii) the gas is in local thermodynamic equilibrium; it is not scattering, a diffuse absorber and emitter with an index of refraction of unity, and (iv) the gas is an optically thick gas so that the Rosseland approximation may be used for the radiative heat transfer term.

The coordinate system is fixed at the plate so that $y=0$ is at the surface of the plate. The plate is initially maintained at a constant temperature $T_{\infty}$ and at time $t=0$ the surface temperature is suddenly increased or decreased to some constant value $T_{w}$ where the subscripts $\infty$ and $w$ refer to the values in the ambient fluid and on the surface of the plate. Under the above assumptions the governing equations for unsteady free convection flow past a vertical flat plate including the effect of radiative heat transfer are as follows

$$
\frac{\partial u}{\partial t}=v \frac{\partial^{2} u}{\partial y^{2}}+g \beta\left(T-T_{\infty}\right),
$$


$\varrho c_{p} \frac{\partial T}{\partial t}=k \frac{\partial^{2} T}{\partial y^{2}}-\frac{\partial q^{r}}{\partial y}$

since we consider a doubly-infinite surface and hence all physical variables are functions of $t$ and $y$ only. Here $u, T$, $v, \varrho, c_{p}, \beta$ and $k$ are the velocity along the plate, the temperature, the kinematic viscosity, the density, the specific heat, the coefficient of thermal expansion and the thermal conductivity of the fluid respectively, and $g$ is the acceleration of gravity.

For a sudden change in surface temperature the initial and boundary conditions pertinent to the problem under consideration are

$t \leqq 0: \quad u=0, \quad T=T_{\infty} \quad$ everywhere

$t>0: \quad u=0, \quad T=T_{w} \quad$ at $y=0$

$u \rightarrow 0, \quad T \rightarrow T_{\infty} \quad$ as $y \rightarrow \infty$.

One sees that the energy Eq. (2) includes the derivation of the radiative heat flux, $q^{r}$. Hence an additional relation is needed to evaluate $q^{r}$. In order to obtain the essential features of the effect of radiative heat transfer, we consider the simple case of an optically thick medium only. For this medium the Rosseland [3] approximation is employed and the radiative heat flux term is given by

$q^{r}=-\frac{16}{3} \frac{\sigma T^{3}}{K_{t}} \frac{\partial T}{\partial y}$

where $\sigma$ is the Stefan-Boltzmann constant for radiative flux and $K_{t}$ the Rosseland mean absorption coefficient. Thus, Eq. (2) may be written as

$\varrho c_{p} \frac{\partial T}{\partial t}=\frac{\partial}{\partial y}\left\{\left(\frac{16}{3} \frac{\sigma T^{3}}{K_{t}}+k\right) \frac{\partial T}{\partial y}\right\}$.

We now introduce the dimensionless variables

$\bar{t}=\frac{t}{t_{0}}, \quad \bar{y}=\frac{y}{L}, \quad \bar{u}=\frac{u}{u_{0}}, \quad \Theta=\frac{T}{T_{\infty}}, \quad \Theta_{w}=\frac{T_{w}}{T_{\infty}}$

where $L$ is a characteristic length of the plate, $t_{0}$ and $u_{0}$ are the time and velocity characteristics which may be conveniently defined as $t_{0}=L^{2} / v$ and $u_{0}=g \beta\left|T_{w}-T_{\infty}\right| L^{2} / v$. Then, Eqs. (1) and (5) become

$\frac{\partial \bar{u}}{\partial \bar{t}}=\frac{\partial^{2} \bar{u}}{\partial \bar{y}^{2}}+\frac{\Theta-1}{\Theta_{w}-1}$,

$\frac{\partial \Theta}{\partial \bar{t}}=\frac{1}{\operatorname{Pr}} \frac{\partial}{\partial \bar{y}}\left\{\left(\frac{4}{3} \frac{\Theta^{3}}{N_{r}}+1\right) \frac{\partial \Theta}{\partial \bar{y}}\right\}$

in which $\operatorname{Pr}$ is the Prandtl number and $N_{r}=k K_{t} / 4 \sigma T_{\infty}^{3}$ is the conduction-to-radiation parameter (radiative flux number). Also the initial and boundary conditions now read

$$
\begin{aligned}
& \bar{t} \leqq 0: \quad \bar{u}=0, \quad \Theta=1 \quad \text { everywhere } \\
& \bar{t}>0: \quad \bar{u}=0, \quad \Theta=\Theta_{w} \quad \text { at } \quad \bar{y}=0 \\
& \bar{u} \rightarrow 0, \quad \Theta \rightarrow 1 \quad \text { as } \quad \bar{y} \rightarrow \infty .
\end{aligned}
$$

Since we consider only the case where the similar solutions of Eqs. (7) and (8) exist, it is convenient to introduce the following similarity variables

$\eta=\frac{\bar{y}}{2 \sqrt{t}}, \quad \bar{u}=\bar{t} f(\eta), \quad \Theta=\Theta(\eta)$.

In terms of new variables (10), Eqs. (7) and (8) may be readily transformed into a set of coupled, second-order ordinary differential equations

$$
\begin{aligned}
& f^{\prime \prime}+2 \eta f^{\prime}-4 f=-4 \frac{\Theta-1}{\Theta_{w}-1}, \\
& \left(1+\frac{4}{3} \frac{\Theta^{3}}{N_{r}}\right) \Theta^{\prime \prime}+\frac{4}{N_{r}} \Theta^{2}\left(\Theta^{\prime}\right)^{2}+2 \operatorname{Pr} \eta \Theta^{\prime}=0
\end{aligned}
$$

with the boundary conditions

$\eta=0: \quad f(0)=0, \quad \Theta(0)=\Theta_{w}$,

$\eta=\infty: \quad f(\infty)=0, \quad \Theta(\infty)=1$

and the primes denote derivation with respect to $\eta$.

\section{Results and discussions}

Let us first mention that Eq. (12) is a nonlinear ordinary differential equation and is not amenable to a closed form nor simple solution as in the case of non-radiative $\left(N_{r} \rightarrow \infty\right)$ free convection flow problem. This equation was derived by Heinisch and Viskanta [4] under a different context. These authors have solved Eq. (12) numerically and presented the results graphically for $\Theta_{w}=1$ only. We will, however, solve here this equation for the entire range of interest of the parameters $\Theta_{w}$ and $N_{r}$, and give the results in the form of tables and graphs too. Before proceeding with numerical solutions of Eqs. (11) and (12) subject to the boundary conditions (13), we seek a perturbation solution of Eq. (12) for large values of $N_{r}$ $(\geqq 1)$.

By expanding $\Theta(\eta)$ in a perturbation series

$\Theta(\eta)=\Theta_{0}(\eta)+\varepsilon \Theta_{1}(\eta)+\varepsilon^{2} \Theta_{2}(\eta)+\ldots$

where $\varepsilon=1 / N_{r}$, Eq. (12) along with the boundary conditions (13) can be separated as

$$
\begin{aligned}
& \varepsilon^{0}: \Theta_{0}^{\prime \prime}+2 \operatorname{Pr} \eta \Theta_{0}^{\prime}=0 \\
& \Theta_{0}(0)=\Theta_{w}, \quad \Theta_{0}(\infty)=1 ; \\
& \varepsilon^{1}: \quad \Theta_{1}^{\prime \prime}+2 \operatorname{Pr} \eta \Theta_{1}^{\prime}=-4\left\{\Theta_{0}^{2} \Theta_{0}^{\prime 2}+\frac{1}{3} \Theta_{0}^{3} \Theta_{0}^{\prime \prime}\right\} \\
& \Theta_{1}(0)=0, \quad \Theta_{1}(\infty)=0 ; \\
& \varepsilon^{2}: \Theta_{2}^{\prime \prime}+2 \operatorname{Pr} \eta \Theta_{2}^{\prime} \\
&=-4\left\{\Theta_{0}^{2} \Theta_{1} \Theta_{0}^{\prime \prime}+\frac{1}{3} \Theta_{0}^{3} \Theta_{1}^{\prime \prime}+2 \Theta_{0} \Theta_{1} \Theta_{0}^{\prime 2}+2 \Theta_{0}^{\prime} \Theta_{1}^{\prime} \Theta_{0}^{2}\right\} \\
& \Theta_{2}(0)=0, \quad \Theta_{2}(\infty)=0 .
\end{aligned}
$$


The solution to the zeroth-order Eqs. (15) is

$\Theta_{0}(\eta)=\Theta_{w}+\left(1-\Theta_{w}\right) \operatorname{erf}(\sqrt{\operatorname{Pr}} \eta)$

where erf $x$ is an error function.

Equations (16) and (17) can be written in the general form

$\Theta_{n}^{\prime \prime}+2 \operatorname{Pr} \eta \Theta_{n}^{\prime}=r_{n}(\eta)$

$\Theta_{n}(0)=0, \quad \Theta_{n}(\infty)=0, \quad n=1,2$

and have the solution expressed as follows

$$
\begin{aligned}
\Theta_{n}(\eta) & =\int_{0}^{\eta} \exp \left(-\operatorname{Pr} \tilde{\eta}^{2}\right) \int_{0}^{\tilde{\eta}} \exp \left(\operatorname{Pr} \tilde{\tilde{\eta}}^{2}\right) r_{n}(\tilde{\tilde{\eta}}) d \tilde{\tilde{\eta}} d \tilde{\eta} \\
- & \left\{\int_{0}^{\infty} \exp \left(-\operatorname{Pr} \eta^{2}\right) \int_{0}^{\eta} \exp \left(\operatorname{Pr} \tilde{\eta}^{2}\right) r_{n}(\tilde{\eta}) d \tilde{\eta} d \eta\right\} \operatorname{erf}(\sqrt{\operatorname{Pr} \eta)}
\end{aligned}
$$

where the $r_{n}$ 's are the right hand sides of (16) and (17).

On te other hand, the linear ordinary differential equations (15) to (17) were integrated numerically for some values of the temperature ratio $\Theta_{w}\left(\equiv T_{w} / T_{\infty}\right)$ in the case of a cold wall. Through the calculations the Prandtl number $P r$ is equal to 0.733 . The accuracy of the perturbation problem has ben studied by comparing the results with those obtained by direct integration of the nonlinear energy Eq. (12). The results for the dimensionless temperature gradient at the wall, $\Theta^{\prime}(0)$, are listed in Table 1 which shows an excellent agreement between the perturbation and exact numerical solutions.

\begin{tabular}{|c|c|c|c|}
\hline$\Theta_{w}$ & $N_{r}$ & Perturbation & Finite-difference \\
\hline \multirow[t]{8}{*}{0.0} & 1 & 1.028584 & 1.0286 \\
\hline & 5 & 0.981501 & 0.9815 \\
\hline & 10 & 0.973966 & 0.9740 \\
\hline & 15 & 0.971374 & 0.9714 \\
\hline & 20 & 0.970062 & 0.9701 \\
\hline & 100 & 0.966873 & 0.9669 \\
\hline & 1000 & 0.966147 & 0.9661 \\
\hline & 10000 & 0.966074 & 0.9661 \\
\hline \multirow[t]{8}{*}{0.25} & 1 & 0.791478 & 0.7915 \\
\hline & 5 & 0.741097 & 0.7411 \\
\hline & 10 & 0.733198 & 0.7330 \\
\hline & 15 & 0.730241 & 0.7302 \\
\hline & 20 & 0.728834 & 0.7288 \\
\hline & 100 & 0.725414 & 0.7254 \\
\hline & 1000 & 0.724636 & 0.7246 \\
\hline & 10000 & 0.724558 & 0.7246 \\
\hline \multirow[t]{8}{*}{0.5} & 1 & 0.512673 & 0.5127 \\
\hline & 5 & 0.492337 & 0.4923 \\
\hline & 10 & 0.487896 & 0.4879 \\
\hline & 15 & 0.486322 & 0.4863 \\
\hline & 20 & 0.485517 & 0.4855 \\
\hline & 100 & 0.483538 & 0.4835 \\
\hline & 1000 & 0.483084 & 0.4831 \\
\hline & 10000 & 0.483038 & 0.4830 \\
\hline
\end{tabular}

Table 1. Comparison of $\Theta^{\prime}(0)$ obtained by two methods

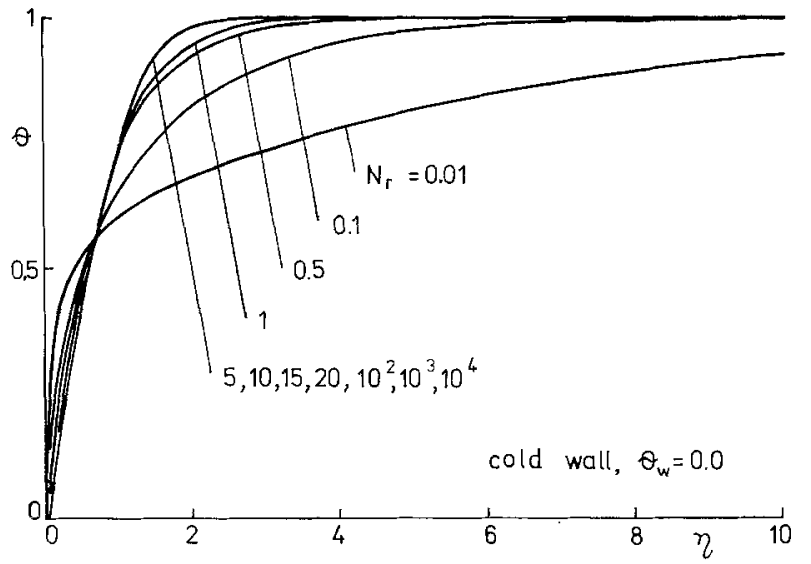

Fig. 1. Effect of radiation on flow temperature distribution for $\Theta_{w}=0.0$

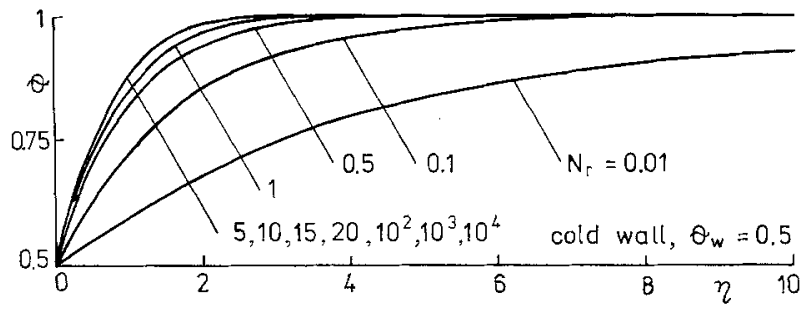

Fig. 2. Effect of radiation on flow temperature distribution for $\Theta_{w}=0.5$

A finite-difference numerical scheme (see $\mathrm{Na}$ [5]) has been used to solve Eqs. (11) and (12) subject to the boundary conditions (13). Here we present some typical results and conclusions. Figures 1 and 2 show the effects of the radiative parameter $N_{r}$ on the function $\Theta(\eta)$ which is directly related to the temperature in the radiative medium for $\Theta_{w}=0.0$ and 0.5 , respectively. From Fig. 1, we see that for $\Theta_{w}=0.0$ the thermal boundary layer becomes thicker as the radiation parameter becomes smaller (i.e. radiation predominant). Contrary, for $\Theta_{w}=0.5$ the effect of $N_{r}$ on the temperature field is just opposite, Fig. 2. As $N_{r}$ gets larger, the radiation component plays less of a role, radiation would affect the conduction of heat to a lesser extent. It is also important to note that there is a no detectable difference in the temperature profile for $N_{r}=5$ and greater, hence the curve for $N_{r}=5$ may be considered as characterizing the non-radiative case. But, as $N_{r}$ decreases the temperature profile has greater changes in curvature, particularly near the wall.

Figure 3 shows the effects of variable wall temperature $\Theta_{w}$ on the temperature profile by keeping the radiative flux number constant and equal to 5. The thermal boundary layer appears to become thicker for the hot wall and thinner for the cold wall as $\Theta_{w}$ increases.

Since the effect of radiation upon the velocity field is small and to conserve space the results concerning velocity profiles are omitted in this paper. 


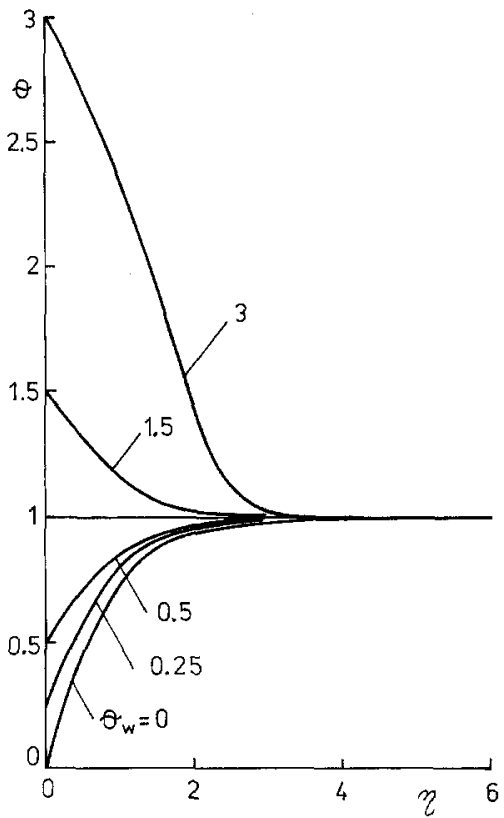

Fig. 3. Effect of wall temperature on flow temperature distribution for $N_{r}=5$

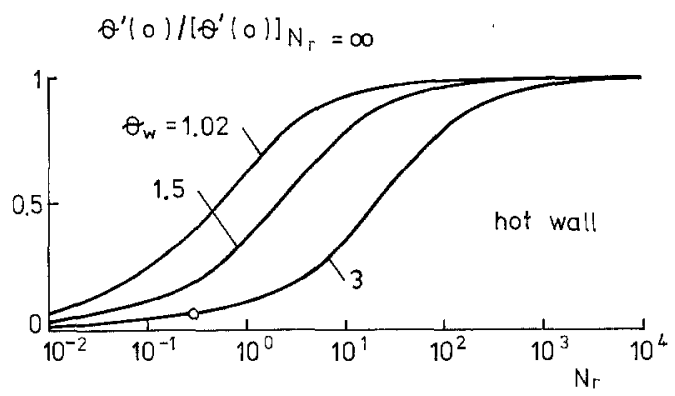

Fig. 4. Variation of temperature gradient ratio at the wall

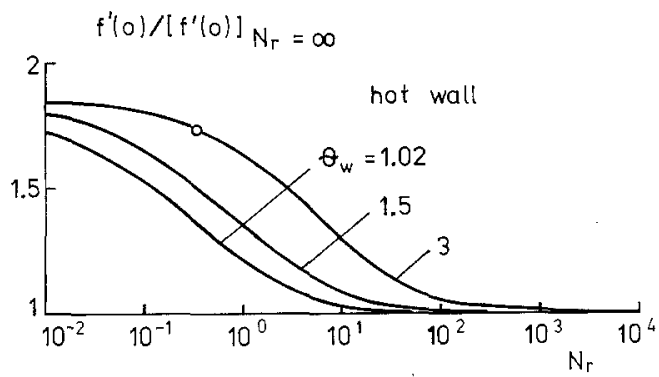

Fig. 5. Variation of velocity gradient ratio at the wall

It is now of interest to delineate the relative contributions of radiation and natural convection to the heat flux from the plate and skin friction on the plate. The heat flux and skin friction are written

$q_{w}=-\left\{\left(\frac{16}{3} \frac{\sigma T^{3}}{K_{t}}+k\right) \frac{\partial T}{\partial y}\right\}_{y=0}, \quad \tau_{w}=\mu\left(\frac{\partial u}{\partial y}\right)_{y=0}$ or in dimensionless form

$\frac{N u_{r}+N u_{c}}{\bar{t}^{1 / 2}}=\frac{1}{2}\left\{\frac{4}{5} \frac{\Theta_{w}^{3}}{N_{r}\left(\Theta_{w}-1\right)}+\frac{1}{\Theta_{w}-1}\right\}\left[-\Theta^{\prime}(0)\right]$

and

$\frac{C_{f}}{\bar{t}^{1 / 2}}=\frac{1}{2} f^{\prime}(0)$,

where $\mu$ is the coefficient of dynamic viscosity, $N u_{r}$ and $N u_{c}$ are, respectively, the radiation and conduction Nusselt numbers.

Restricting our discussion to the hot wall case, Fig. 4 illustrates the variation of the dimensionless temperature gradient at the wall contributed by radiation for a range of $N_{r}$ and under three different $\Theta_{w}$ 's. Results are not included for large values of $\Theta_{w}$ because for small values of $N_{r}$, i.e. radiation dominated situation, the boundary layer becomes very thick resulting in the necessity to integrate to excessive values of the cross-stream coordinate $\eta$. On

Table 2. Values of $\eta_{0}$ and $\bar{x}_{p \max } / 2 G r \bar{t}^{2} \equiv X\left(\eta_{0}, \bar{t}\right)$

\begin{tabular}{|c|c|c|c|c|c|c|c|}
\hline \multicolumn{4}{|c|}{ Cold wall } & \multicolumn{4}{|c|}{ Hot wall } \\
\hline$\Theta_{w}$ & $N_{r}$ & 0 & $X\left(\eta_{0}, \bar{t}\right)$ & $\Theta_{w}$ & $N_{r}$ & 0 & $X\left(h_{0}, \bar{t}\right)$ \\
\hline \multirow[t]{11}{*}{0.0} & 0.01 & 0.6392 & 0.0880 & \multirow[t]{11}{*}{1.02} & 0.01 & 0.8835 & 0.1960 \\
\hline & 0.1 & 0.4621 & 0.0639 & & 0.1 & 0.6260 & 0.1396 \\
\hline & 0.5 & 0.3864 & 0.0581 & & 0.5 & 0.4800 & 0.0959 \\
\hline & 1 & 0.3727 & 0.0572 & & 1 & 0.4356 & 0.0815 \\
\hline & 5 & 0.3595 & 0.0565 & & 5 & 0.3777 & 0.0630 \\
\hline & 10 & 0.3581 & 0.0564 & & 10 & 0.3681 & 0.0598 \\
\hline & 15 & 0.3676 & 0.0564 & & 15 & 0.3642 & 0.0587 \\
\hline & 20 & 0.3573 & 0.0564 & & 20 & 0.3621 & 0.0581 \\
\hline & 100 & 0.3568 & 0.0563 & & 100 & 0.3576 & 0.0567 \\
\hline & 1000 & 0.3566 & 0.0563 & & 1000 & 0.3567 & 0.0563 \\
\hline & 10000 & 0.3566 & 0.0563 & & 10000 & 0.3566 & 0.0563 \\
\hline \multirow[t]{11}{*}{0.25} & 0.01 & 0.6542 & 0.1137 & \multirow[t]{11}{*}{1.5} & 0.01 & 1.0274 & 0.2169 \\
\hline & 0.1 & 0.4885 & 0.0758 & & 0.1 & 0.7385 & 0.1714 \\
\hline & 0.5 & 0.4019 & 0.0621 & & 0.5 & 0.5555 & 0.1240 \\
\hline & 1 & 0.3818 & 0.0595 & & 1 & 0.4915 & 0.1039 \\
\hline & 5 & 0.3620 & 0.0570 & & 5 & 0.3968 & 0.0715 \\
\hline & 10 & 0.3591 & 0.0567 & & 10 & 0.3779 & 0.0646 \\
\hline & 15 & 0.3583 & 0.0565 & & 15 & 0.3717 & 0.0620 \\
\hline & 20 & 0.3579 & 0.0565 & & 20 & 0.3681 & 0.0606 \\
\hline & 100 & 0.3569 & 0.0563 & & 100 & 0.3586 & 0.0572 \\
\hline & 1000 & 0.3566 & 0.0563 & & 1000 & 0.3568 & 0.0564 \\
\hline & 10000 & 0.3566 & 0.0563 & & 10000 & 0.3566 & 0.0563 \\
\hline \multirow[t]{11}{*}{0.5} & 0.01 & 0.7034 & 0.1466 & \multirow[t]{11}{*}{3} & 0.01 & 1.3009 & 0.2376 \\
\hline & 0.1 & 0.5187 & 0.0944 & & 0.1 & 1.0028 & 0.2157 \\
\hline & 0.5 & 0.4212 & 0.0697 & & 0.5 & $\begin{array}{l}\text { solution } \\
\text { converg }\end{array}$ & does not \\
\hline & 1 & 0.3952 & 0.0639 & & 1 & 0.6908 & 0.1643 \\
\hline & 5 & 0.3660 & 0.0580 & & 5 & 0.5044 & 0.1129 \\
\hline & 10 & 0.3608 & 0.0572 & & 10 & 0.4472 & 0.0934 \\
\hline & 15 & 0.3593 & 0.0569 & & 15 & 0.4211 & 0.0841 \\
\hline & 20 & 0.3586 & 0.0568 & & 20 & 0.4074 & 0.0786 \\
\hline & 100 & 0.3570 & 0.0564 & & 100 & 0.3675 & 0.0617 \\
\hline & 1000 & 0.3566 & 0.0563 & & 1000 & 0.3575 & 0.0569 \\
\hline & 10000 & 0.3566 & 0.0563 & & 10000 & 0.3567 & 0.0564 \\
\hline
\end{tabular}


the other hand, for $N_{r}=0.5$ the calculations show a singularity at $\Theta_{w}=3$. In fact, this is in accordance with the observation from [6] that the Rosseland approximation for the radiative heat flux breaks down in the vicinity of the plate. An inspection of the figure reveals that the parameter $N_{r}$ has considerable influence on the heat flux at the wall, i.e. $y=0$ or $t=\infty$, steady state flow. But, since the larger $N_{r}$ the smaller is the role radiation plays on the conduction heat flux.

Similarly, the variation of the dimensionless velocity gradient at the wall with $N_{r}$ and three different $\Theta_{w}$ 's is shown in Fig. 5. An opposite trend to the heat flux ratio is observed for the fraction of skin friction.

Further, the dimensionless penetration distance of the fluid originally at the leading edge of a semi-infinite flat plate can be computed from, see for instance [7]

$\frac{\bar{x}_{p}}{2 G r \vec{t}^{2}}=\eta^{4} \int_{\eta}^{\infty} f(\bar{\eta}) \frac{d \tilde{\eta}}{\tilde{\eta}^{5}}$

where $G r=g \beta\left|T_{w}-T_{\infty}\right| L^{3} / v^{2}$ is the Grashof number. If $\eta_{0}$ denotes the root of the equation

$\frac{\partial \bar{x}_{p}}{\partial \eta}=0$

then, the dimensionless maximum penetration distance is given as

$\bar{x}_{p \max }=\bar{x}_{p}\left(\eta_{0}, \bar{t}\right)$.

Numerical calculation of the values of $\eta_{0}$ and $\bar{x}_{p \max }$ was performed for a number of combinations of $\Theta_{w}$ and $N_{r}$.
Some of the results are given in Table 2. It is seen from this table that the penetration distance increases with the increase of the wall temperature $\Theta_{w}$ and decreases with the increase of the radiation parameter $N_{r}$ to the values of non-radiative free convection problem.

\section{References}

1. Pai, S. I.; Scaglione, A. P.: Appl. Sci. Res. 22 (1970) 97

2. Cheng, E. H.; Özisik, M. N.: Appl. Sci. Res. 28 (1973) 185

3. Rosseland, S.: Astrophysik auf Atom-Theoretischer Grundlage. Berlin: Springer 1931

4. Heinisch, R. P.; Viskanta, R.: AIAA Jl. 6 (1968) 1409

5. Na, T. Y.: Computational methods in engineering boundary value problems. New York: Academic Press 1979

6. Macken, N. A.; Hartnett, J. P.: Wärme- Stoffübertrag. 6 (1973) 205

7. Mizukami, K.: Int. J. Heat Mass Transfer 20 (1977) 981

I. Pop

Faculty of Mathematics

University of Cluj

R-3400 Cluj, CP 253, Romania

T. Y. Na

Department of Mechanical Engineering

University of Michigan-Dearborn

Michigan 48128

Received March 14, 1983 